\title{
Design of Transcutaneous Electrical Nerve Stimulating Machine Using 555 Timer and 7555 Timer
}

\author{
Soumyadeep Sarkar \\ Department of Electrical and Electronic Engineering (EEE), Kurukshetra University, Kurukshetra
}

\begin{abstract}
Transcutaneous Electrical Nerve Stimulation or TENS is an electrotherapeutical process of using electrical current to excite and stimulate the nervous system of a living body. The term 'electrotherapy' has applied to a variety of treatments including the use of machines such as the deep brain stimulators for neural diseases, shock therapy, etc. This term has also been applied focussing to the use of electrical current to speed up the process of wound healing. Additionally it is a non pharmacological treatment and falls into the category of alternative cures and medicines. TENS devices are used as a non- invasive nerve stimulation system designed to reduce both acute and chronic pain by the application of electrical current through electrodes placed on the skin for pain control. This paper documents the design, operation and construction of such a system which can be used to treat ailments at a very low cost and can also be switched by the user from a low frequency system to a high frequency system for desired application
\end{abstract}

Keywords: TENS Machine; electrotherapy; 555 Timer IC; 7555 Timer IC; IRF9640 MOSFET; Intensity control; Square Wave Generator; Variable frequency

\section{Introduction}

A Transcutaneous Electrical Nerve Stimulating Machine or the TENS Machine is device which uses electrical current at certain frequencies to reduce acute and chronic pain by exciting and stimulating the peripheral nervous system of the human body by means of two electrodes connected to the surface of the body.

This typical battery-operated TENS device can modulate pulse width, frequency and intensity. It can be applied with variable frequencies, from low $(<10 \mathrm{~Hz})$ to high $(>50 \mathrm{~Hz})$. Intensity can also be altered from sensory intensities into motor intensities. Sensory intensity is when the patient feels a strong but comfortable sensation without motor contraction. High intensity usually involves a motor contraction but is not painful. Basically, higher-frequency stimulation is delivered at sensory intensity, and lowfrequency stimulation is delivered at motor intensity.

Similarly, a few studies have shown evidence that TENS may modulate or suppress pain signals in the brain by using evoked cortical potentials to show that electric stimulation of peripheral A-beta sensory fibres reliably suppressed A-delta fiber nociceptive processing.

Some studies showed that, regardless of intensity, different frequencies activate central mechanisms to produce analgesia. Specifically, low-frequency TENS activates $\mu$ opioid receptors in the spinal cord and the brainstem which helps the body produce its own endorphins and increase local blood flow to relieve pain, whereas high frequency TENS activates $\delta$-opioid receptors in the spinal cord and the brainstem which further blocks the pain impulses going to the brain.

Related studies have investigated the principle role of serotoninergic, noradrenergic, muscarinic and $\gamma$ - aminobutyric acid systems on the analgesia produced by both high frequency and low frequency nerve stimulation.

Using functional magnetic resonance imaging (fMRI), one study investigated that high-frequency TENS showed a decrease in pain-related cortical activations in patients with carpal tunnel syndrome, while the other showed that lowfrequency TENS decreased shoulder impingement pain and modulated pain-induced activation in the brain.

A head-mounted TENS device called Cefaly was first approved by the United States Food and Drug Administration, on March 11, 2014, for the prevention of severe headache. The Cefaly device was found effective in prevention of migraine attacks in randomized placebocontrolled trials. This was the first TENS device which the FDA approved for pain prevention.

The device described in this paper uses a 555 timer to create square waves. The reason square waves are used because the Square wave permits the frequencies to penetrate the body more efficiently at any given $\mathrm{kHz}$ output. Square waves are considered the superior "frequency to wave" output for any frequency generating device or machine.

\section{A. What is a Square Wave?}

A square wave is a non-sinusoidal periodic waveform (can be represented as an infinite summation of sinusoidal waves), where the amplitude alternates at a steady frequency between fixed minimum and maximum values, with the same duration at minimum and maximum. The transition between minimum to maximum is instantaneous for an ideal square wave though this is not realizable in physical systems. 
International Journal of Science and Research (IJSR)

ISSN (Online): 2319-7064

Index Copernicus Value (2015): 78.96 | Impact Factor (2015): 6.391

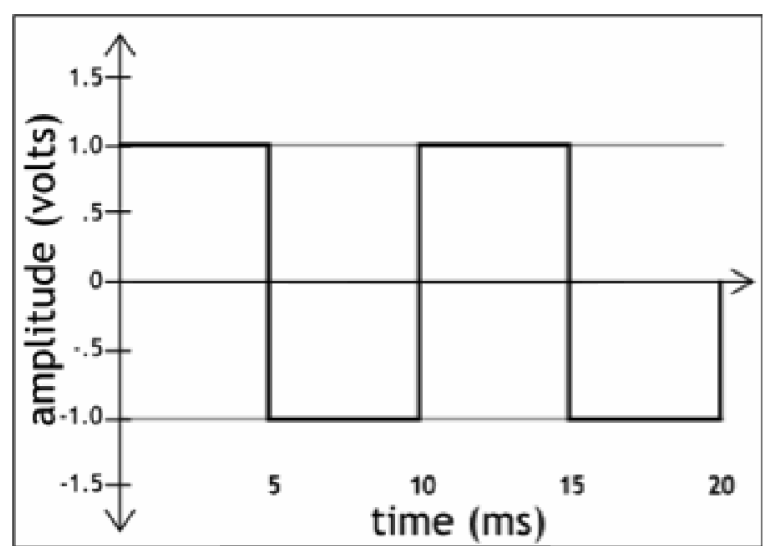

Figure 1: Square Wave

Square waves are universally encountered in digital switching circuits and are naturally generated by binary (two-level) logic devices. Its stochastic counterpart is a twostate trajectory. A similar but not necessarily symmetrical wave, with arbitrary durations at minimum and maximum, is called a pulse wave. They are generally used as timing references or "clock signals", because of their fast transitions and are relatively suitable for triggering synchronous logic circuits at deliberately precise determined intervals.

However, as the frequency-domain graph displays, square waves contain a wide range of harmonics and these can create electromagnetic radiation or pulses of current that can interfere with other nearby circuits ipso facto causing noise or errors in the system. The square wave can be defined mathematically in many numbers of ways such as;

It can be defined as simply the sign function of a periodic function, an example being a sinusoid:

$$
\begin{aligned}
& x(t)=\operatorname{sgn}(\sin [t]) \\
& v(t)=\operatorname{sgn}(\cos [t])
\end{aligned}
$$

which will be 1 when the sinusoid is positive. When the sinusoid is negative, it will be -1and at the discontinuities it will be 0 .

A square wave can also be defined with respect to the Heaviside step function $u(t)$ or the rectangular function $\Pi(t)$ :

$$
x(t)=\sum_{n=-\infty}^{+\infty} \Pi(t-n T)=\sum_{n=-\infty}^{+\infty}\left(u\left[t-n T+\frac{1}{2}\right]-u\left[t-n T-\frac{1}{2}\right]\right)
$$

$T$ is 2 for a $50 \%$ duty cycle. It can also be defined in a hybrid way:

$$
x(t)= \begin{cases}1, & |t|<T_{1} \\ 0, & T_{1}<|t| \leq \frac{1}{2} T\end{cases}
$$

when,

$$
x(t+T)=x(t)
$$

In terms of sine and cosecant with period $p$ and amplitude $a$ :

$$
y(x)=a \times \csc \left(\frac{2 \pi}{p} x\right)\left|\sin \left(\frac{2 \pi}{p} x\right)\right|
$$

A square wave can also be generated using the floor function in the following two ways. Namely, directly and indirectly: Directly:

$$
y(x)=m(2\lfloor\nu x\rfloor-\lfloor 2 \nu x\rfloor+1)
$$

Indirectly:

$$
y(x)=m(-1)^{\lfloor\nu x \mid}
$$

Where, ' $m$ ' is the magnitude and ' $v$ ' is the frequency.

Note: $y=a \tan \sin x+a \cot \sin x$

Now, it can also be represented in terms of Fourier analysis;

It can be expressed as the limiting case of an infinite series of sinusoidal waves, with fast convergence. Using, Fourier expansion with cycle frequency $(f)$ over time $(t)$, we can represent an ideal square wave with amplitude of 1 as an infinite series of the form:

$$
\begin{aligned}
x_{\text {square }}(t) & =\frac{4}{\pi} \sum_{k=1}^{\infty} \frac{\sin (2 \pi(2 k-1) f t)}{2 k-1} \\
& =\frac{4}{\pi}\left(\sin (2 \pi f t)+\frac{1}{3} \sin (6 \pi f t)+\frac{1}{5} \sin (10 \pi f t)+\cdots\right)
\end{aligned}
$$

The ideal square wave contains only components of odd integer harmonic frequencies.

An ideal mathematical square wave changes between the high states and the low states instantaneously and it changes without under shooting or over shooting. This is impossible to achieve in physical systems, as it would require infinite bandwidth. If the system is over damped, then the waveform will never actually reach the theoretical high levels and low levels, and if the system is under damped, it will oscillate about the high levels and low levels before settling down.

\section{B. Using of 555 and 7555 Timer IC to generate Square Wave}

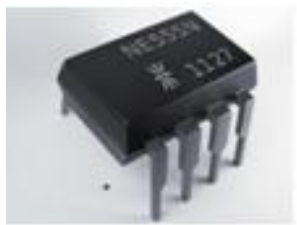

(a)

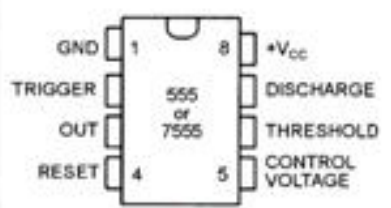

(b)
Figure 2: (a), (b): 555 - 7555 Chip

The 555 timer IC was designed by an American Company named Signetics in the year 1971. The 555 and the 7555 Oscillator IC is a type of relaxation oscillator for generating stabilized square wave output waveforms of either a fixed frequency of up to $500 \mathrm{kHz}$ or of varying duty cycles from 50 to $100 \%$. A true square wave has a $50 \%$ duty cycle equal high and low periods. The average level of a square wave is also given by the duty cycle, so by varying the on and off periods and then averaging, it is possible to represent any value between the two limiting levels. This is the basis of pulse width modulation.

It is an integrated circuit (chip) having a variety of uses as a timer, flip-flop element, pulse generator or even in oscillator applications. The 7555 IC mentioned in this project is nothing but the low power version of 555 IC and it works exactly the same with little less noise and increased efficiency. The 7555 timer mentioned in this project is a CMOS RC low-power devices offer significant performance over the standard 555 bipolar timer. It is a stable controller capable of producing accurate time delays or frequencies. In the one-shot mode or monostable operation, the pulse width of each circuit is precisely controlled by one external resistor 


\section{International Journal of Science and Research (IJSR) \\ ISSN (Online): 2319-7064 \\ Index Copernicus Value (2015): 78.96 | Impact Factor (2015): 6.391}

and capacitor. It causes less supply noise than the classic 555 and it usually does not require a "control" capacitor and in many cases does not require a decoupling capacitor on the power supply.

$50 \%$ Duty Cycle Frequency Equation is given by:

$$
\begin{array}{r}
f=\frac{1}{0.693\left(2 R_{2}\right) \cdot C} \\
\text { Where, } \mathrm{f}=\text { frequency, } \\
\mathrm{R}=\text { Resistance, } \\
\mathrm{C}=\text { Capacitance }
\end{array}
$$

The re-triggering is basically achieved by connecting the trigger input (pin 2) and the threshold input (pin 6) together, allowing the device to act as an astable oscillator. However, the 555 Oscillator has no stable states as it continuously switches from one state to the other. The circuit given below is a basic Astable 555 Oscillator Circuit:

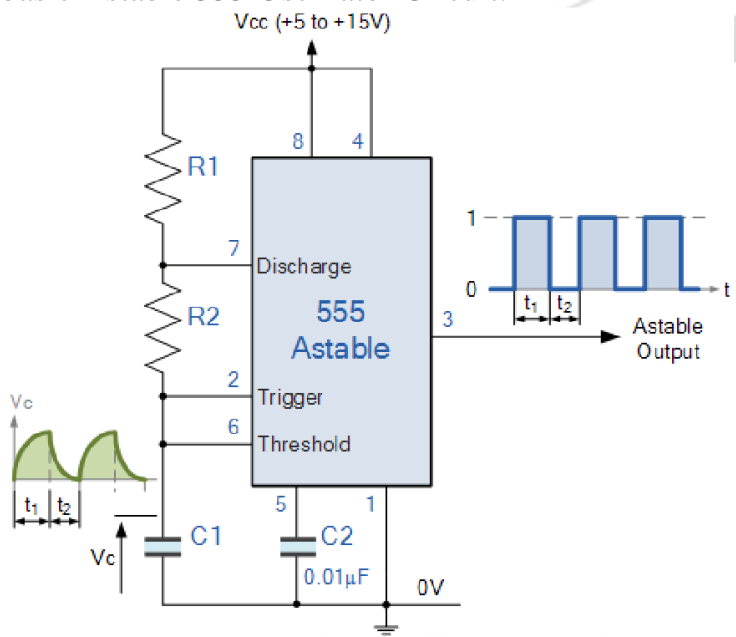

(a)

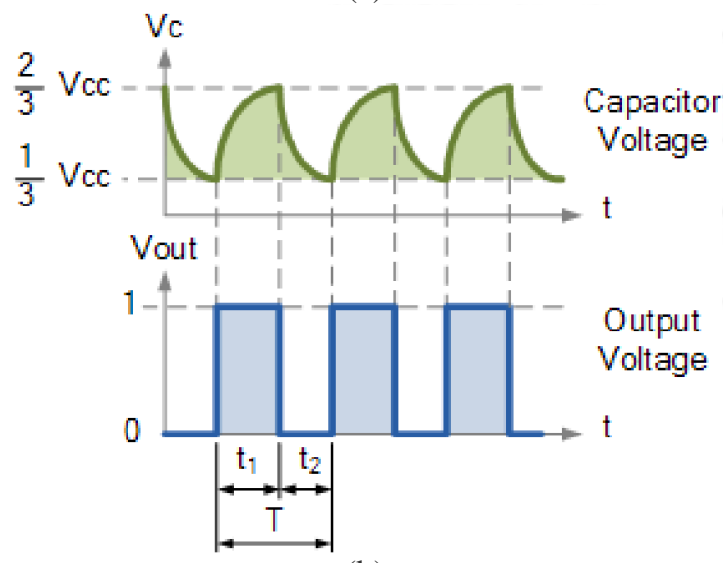

(b)

Figure 3: (a), (b) Astable 555 Oscillator Circuit.

In this 555/ 7555 Oscillator circuit given above, pin 2 and pin 6 are connected together allowing the circuit to re-trigger itself on each and every cycle so it to operate as a free running oscillator. During each cycle capacitor, C charges up through both timing resistors, R1and R2 but discharges itself only through resistor, R2 as the other side of R2 is connected to the discharge terminal, pin 7.
The duration of one full timing cycle is equal to the sum of the two individual times, the capacitor charges and discharges, adding it together and it is given by:

\section{Oscillator Cycle Time:}

$$
T=t_{1}+t_{2}=0.693\left(R_{1}+2 R_{2}\right) . C
$$

The output frequency can be found by inverting the equation above for the total cycle time giving a final equation for the output frequency of the 555 Oscillator as:

\section{Oscillator Frequency Equation:}

$$
f=\frac{1}{T}=\frac{1.44}{\left(R_{1}+2 R_{2}\right) \cdot C}
$$

By manipulating the time constant of just one of the RC combinations, the Duty Cycle also known as the "Mark-toSpace" ratio of the output waveform can be accurately set and is given as the ratio of resistor R2 to resistor R1. The Duty Cycle for the 555 Oscillator or ratio of the "ON" time divided by the "OFF" time is given by:

$$
\begin{aligned}
& \text { Oscillator Duty Cycle: } \\
& \text { Duty Cycle }=\frac{T_{O N}}{T_{O F F}+T_{O N}}=\frac{R_{1}+R_{2}}{\left(R_{1}+2 R_{2}\right)} \%
\end{aligned}
$$

To end, the 555 Oscillator can also be used in a wide range of waveform generator circuits and applications that require very less output current such as in electronic testing devices for creating a whole new range of different output test frequencies. The 555 can be used to provide time delays, as an oscillator, and as a flip-flop element. Certain IC derivatives can provide up to four timing circuits in one package and is still in widespread use because of its affordability and device stability.

\section{Designing the Probes for this Unit}

The probe pads of this unit can be made using simple stiff copper wires coiled and connected to a Silver foil plate and then again covering the plate using another layer of the same foil. Then the silver foil covered pads to be placed on the body. To increase conductivity hair gels can be used or to be more, professional, electrode conducting gels are easily available which are commonly used on ECG machine probes.

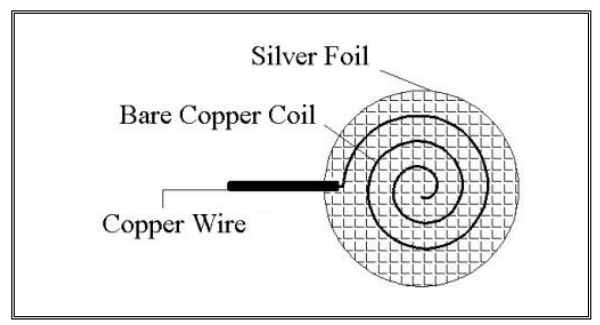

Figure 4: Aluminium Foil Probe Design

Electrode Conductive Gel is extremely useful when electrodes are used to process electrical activity on the skin. The outside layer of the skin is mainly comprised of dead skin cells which in turn will reduce efficiency of the

\section{Volume 5 Issue 11, November 2016




\section{International Journal of Science and Research (IJSR) \\ ISSN (Online): 2319-7064 \\ Index Copernicus Value (2015): 78.96 | Impact Factor (2015): 6.391}

machine. As described above, hair gels can be used but they are not a good alternative as compared to the Electrode Conducting Gels. To add, it is advised that the positive and the negative conducting pads must be kept apart from each other to prevent shorting of the circuit.

\section{The Schematic}

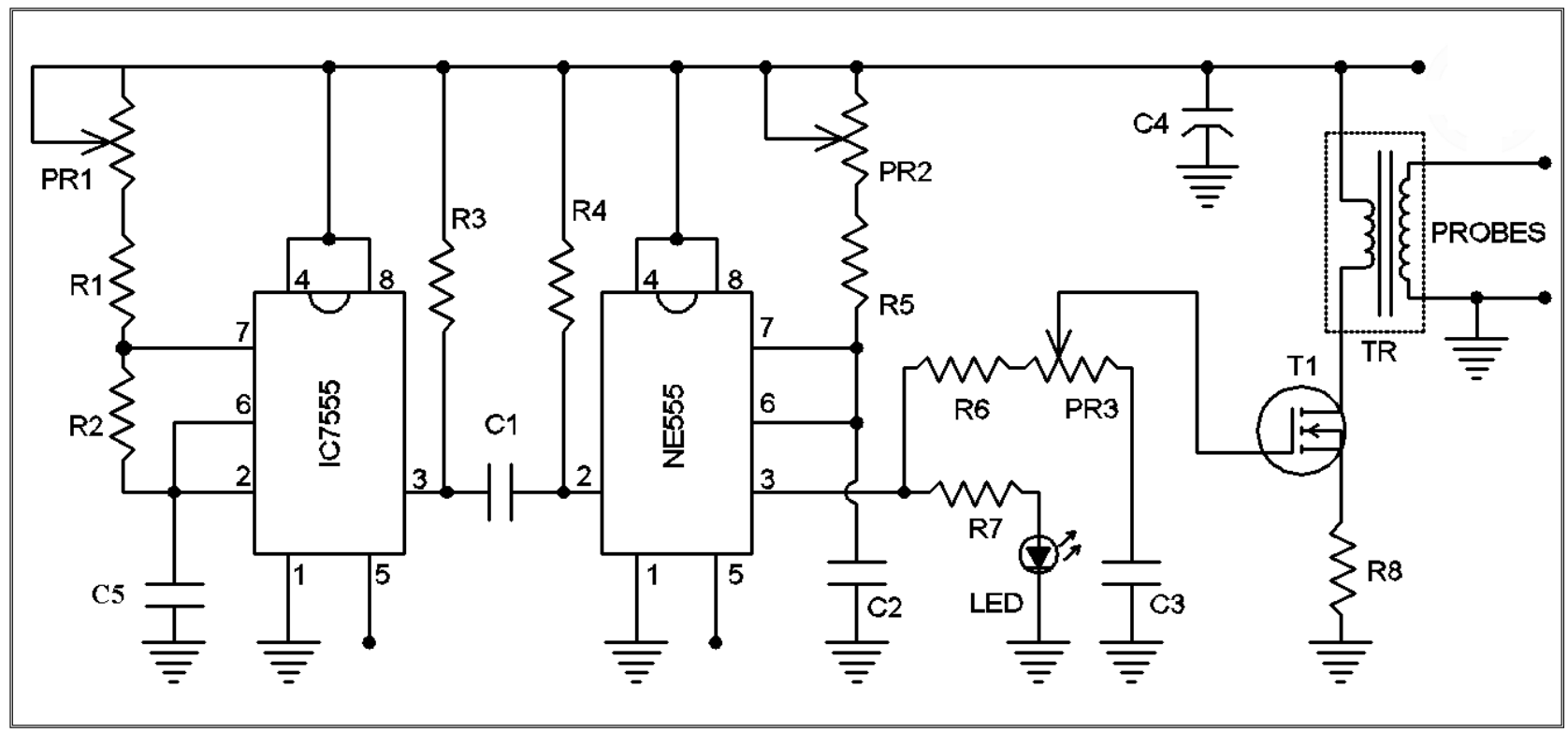

Figure 5: TENS Circuit Diagram

\section{List of Components}

The following table provides a list of components necessary to build the proposed TENS Unit:

Table 1: Component List

\begin{tabular}{|c|c|c|c|c|}
\hline \multicolumn{5}{|c|}{ COMPONENT LIST FOR TENS CIRCUIT } \\
\hline S. No. & Component & Component Type & Values & Comments \\
\hline 1. & PR1 & Potentiometer & $1 \mathrm{M} \Omega$ & $1.4 \mathrm{~Hz}-100 \mathrm{~Hz}$ \\
\hline 2. & PR2 & Potentiometer & $25 \mathrm{k} \Omega$ & 40 uS -270 us \\
\hline 3. & PR3 & Potentiometer & $5 \mathrm{k} \Omega$ & Output Drive \\
\hline 4. & R1 & Resistor & $10 \mathrm{k} \Omega$ & \\
\hline 5. & $\mathrm{R} 2$ & Resistor & $2.2 \Omega$ & \\
\hline 6. & R3 & Resistor & $10 \mathrm{k} \Omega$ & \\
\hline 7. & R4 & Resistor & $100 \mathrm{k} \Omega$ & \\
\hline 8. & R5 & Resistor & $400 \Omega$ & \\
\hline 9. & R6 & Resistor & $100 \Omega$ & \\
\hline 10. & R7 & Resistor & $1 \mathrm{k} \Omega$ & \\
\hline 11. & R8 & Resistor & $22 \mathrm{k} \Omega$ & Min. 3 Watt \\
\hline 12. & $\mathrm{C} 1$ & Capacitor & $1 \mathrm{nF}$ & \\
\hline 13. & $\mathrm{C} 2$ & Capacitor & $10 \mathrm{nF}$ & \\
\hline 14. & $\mathrm{C} 3$ & Capacitor & $10 \mathrm{nF}$ & \\
\hline 15. & $\mathrm{C} 4$ & Capacitor & $100 \mu \mathrm{F}, 16 \mathrm{~V}$ & For Rectifying \\
\hline 16. & $\mathrm{C5}$ & Capacitor & $1 \mu \mathrm{F}$ & \\
\hline 17. & 7555 & IC & & \\
\hline 18. & NE555 & IC & & \\
\hline 19. & T1 & MOSFET & IRF9640 & \\
\hline 20. & LED & Red LED & & Indicator \\
\hline 21. & TR & Transformer & $8 \Omega$ (impedance) & Audio Trans. \\
\hline 22. & IN1 & Input System & $12 \mathrm{VDC}, 500 \mathrm{~mA}$ & \\
\hline
\end{tabular}

\section{A Brief Explanation about the Schematic}

The above mentioned schematic is of the Transcutaneous Electrical Nerve Stimulating Machine, the frequency and the pulse width of which can be varied and controlled according to the desired output by the user. The schematic comprises of two ICs, namely, 7555IC and NE555IC, 7555IC is nothing but the low power version of 555 IC and it works exactly the same with little less noise and increased efficiency; an IRF9640 MOSFET, a MOSFET is recommended over an ordinary transistor is because an ordinary transformer will result to a low power output; 2 potentiometers, one specially for controlling the output drive (5K) and must be set just to feel the pulses lightly and the other potentiometer is for setting the pulse width (PR2). This circuit also consists of a combination of capacitors and one capacitor particularly set to define the output frequency coupled up with the variable resistor or the potentiometer.

A transformer is also used in the schematic which is a small audio transformer with the 8 ohm impedance winding connected to the MOSFET and the larger winding connected to the probe and ground. Using of a "Mode" Transformer is highly recommended. The Turns ratio of the Mode Transformer determines the voltage output. Using of a small 6, 9 or 12 volt power transformer will not cause a big issue, but it will not give a good stabilised output when subjected to a long term use. The input voltage required for this circuit is 9VDC-12VDC and when the output is set at maximum, the output pulses will be of 120 Volts approximately. The output current can be adjusted by adjusting the duty width.

\section{Working}

This TENS machine is designed to work in two ways:

On a high pulse rate of $90-130 \mathrm{~Hz}$ (the normal method of use), the electrical impulses generated by the TENS machine at this frequency interfere and block pain messages sent to the brain. This is due to the gate control theory of pain.

\section{Volume 5 Issue 11, November 2016




\section{International Journal of Science and Research (IJSR) \\ ISSN (Online): 2319-7064}

Index Copernicus Value (2015): 78.96 | Impact Factor (2015): 6.391

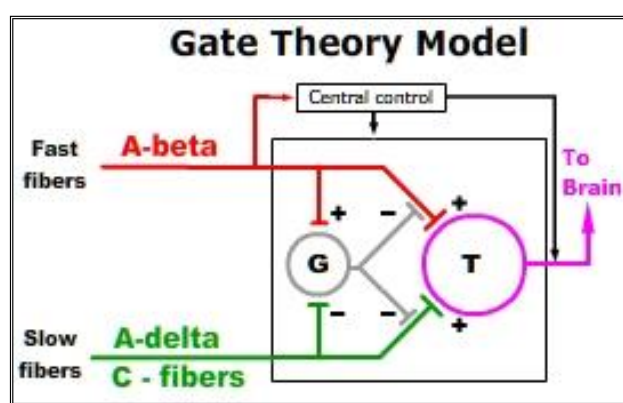

(a)

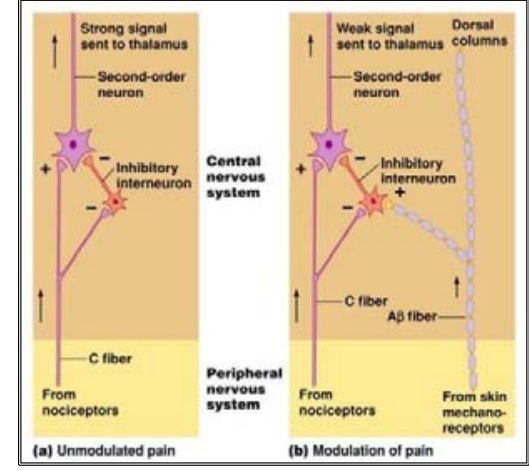

(b)

Figure 6: (a), (b) Gate Theory.

This delivers a theory that there is a gate mechanism in the central nervous system. When the gate is open, pain messages get travels to the brain and we feel pain. When the gate is closed, these pain messages are blocked and we do not feel pain. TENS machines are designed to stimulate certain non-nociceptive fibres and close the gate. In effect, the brain is busy dealing with the messages it receives quickly from the TENS machine, rather than the slower (more painful) pain signals that the body is receiving from somewhere else. This explains why, when an injury is encountered, rubbing that area can temporarily reduce the pain.

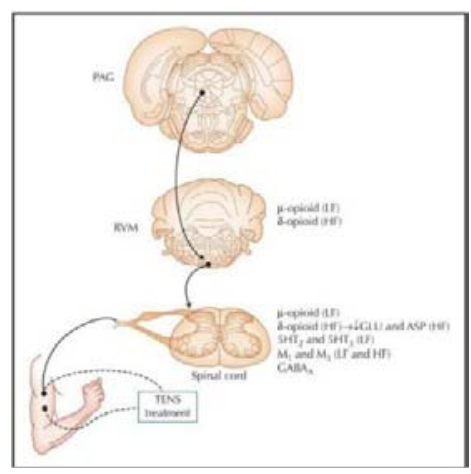

Figure 7: Schematic diagram showing potential pathways activated by low-frequency or high frequency TENS

When the machine is set on a low pulsing, it stimulates the body to make its own pain decreasing chemicals called endorphins. Endorphins are basically the endogenous opioid neuropeptides present in a living being. These are produced by the central nervous system and the pituitary gland. Endorphins are among one of the brain chemical (homologous to the activity of the corticosteroid category of bio-chemicals) known as neurotransmitters, whose function to transmit electrical signals within the nervous system. They are natural pain killers and they act like morphine to maintain analgesia. Additionally, they also make us feel better, improve our mood, increase pleasure.

\section{Applications}

TENS devices are used as a non- invasive nerve stimulation system designed to reduce both acute and chronic pain by the application of electrical current through electrodes placed on the skin for pain control.

The site of application of electrodes of TENS machine is typically near the site of injury. It is possible that application outside the site may also be effective, as central mechanisms can be activated by TENS.

A TENS machine can be personally controlled by the user. The settings can be adjusted, without the need of a medical professional. Typically, a machine would be used for 15-20 minutes per session, several times per day. As far as the applications for personal use is concerned, TENS machines are used to reduce pain from muscular trauma, joints and nerve algesia, musculoskeletal pain. They may work better for these problems than for abdominal, pectoral or head pains. Unlike a lot of medication there are virtually no sideeffects of a TENS machine. However, pregnant females, person with an artificial pacemaker, having episodes of epileptic seizures must not use this machine. The reason, a person having an artificial pacemaker and epilepsy must not use this machine because, the artificial pacemakers implanted into the skin provides artificial impulses to the heart for beating and it also contains very sensitive operational circuitry, using tens with interfere with the electrical activity of the pacemakers, ipso facto causing a malfunction and as far epilepsy is concerned, they are basically caused due to abnormal excessive or synchronous neuronal activity in the brain. So a person having a history of epileptic seizure must refrain from using a TENS, as because it can worsen the condition of the patient.

TENS machines can be used solitarily for pain relief or can be combined with other professional treatments.

The use of a TENS machine might allow reduction of the amount of painkilling medicines people usually take, for pain relief which further involves many side effects. A TENS machine usually have no side effects.

A TENS machine might be worth a trial, particularly if conventional pain relief methods have been tried and no good came out of it.

\section{Instructions of Using A Tens Machine}

1) This TENS machines is specifically crafted and designed so that the user can move around with them working. The machine can be tucked into a pocket or it can be clipped on to a belt.

2) Before placing the electrode pads on the skin, it must be made sure that the machine is switched off.

3) The machine can be tested by holding the pads between the fingers and then carefully turning it on. A tingling sensation must be felt. This will ensure that the machine is working correctly.

\section{Volume 5 Issue 11, November 2016}




\section{International Journal of Science and Research (IJSR) \\ ISSN (Online): 2319-7064 \\ Index Copernicus Value (2015): 78.96 | Impact Factor (2015): 6.391}

4) It must be made sure that the skin, where the electrodes are to be put, must be clean and dry and there are no cuts, scratches or area of skin irritation.

5) The pads must be placed on the either side of the pain. Self-adhesive pads or flexible rubber pads must be used which should be completely layered, on the skin and the pads must be applied, with a thin layer of electrode conductive gel. The two pads should not be applied close to each other. A distance of at least an inch must be maintained.

6) The machine must be turned on slowly, by setting the pulse rate to the required setting and turning it up until a gradual tingling sensation is felt. The sensation needs to be quite strong but not so much that it makes the user uncomfortable.

7) After a few minutes the sensation will start to fade away slightly. This is called accommodation. When this happens, the machine must be turned up slightly and then left for the rest of the time in use. The machine must not be turned up too high, as this can cause over-stimulation which may worsen the condition.

8) At the end of the session the machine must be turned off and the electrodes from the machine must be disconnected.

Note: The machine must be used for at least 45 minutes but can be used for up to 15 hours before the electrodes and the probes need to be cleaned and re-sited.

\section{Cautions When Using A Tens Unit}

1) Electrode pads or probes must not be used on broken skin.

2) Electrodes must not be placed over the front or side of the neck, too close to eyes or in the mouth.

3) The unit must not be used over areas of reduced sensation.

4) The machine must not be used near water such as in the bath or shower. This may lead to a short circuitry which may further damage the circuitry.

5) TENS must not be used while driving or operating heavy machinery.

\section{Conclusion}

Some basic scientific evidence suggests that there are peripheral and central nervous system mechanisms underlying the analgesic action of TENS and both pharmacologic and non pharmacological strategies can be implemented to prevent tolerance caused due to continuous use of this device. Experimental pain studies and clinical trials are beginning to refine parameters of stimulation to obtain the best analgesic function. It also shows that stimulation intensity is a critical factor for the effectiveness of the stimulation.

Recently, two studies showed that application of TENS to the contra lateral hind limb reduces hyperalgesia of the inflammation of the injured limb. Furthermore when hyperalgesia developed bilaterally after a unilateral injury, use of either high frequency or low frequency TENS to the injured area reduced the hyperalgesia bilaterally. In a different model, Somers DL and Clemente FR investigated the sites of electrode placement that would stop the development of allodynia in a chronic constriction injury to the rat sciatic nerve. Studies also showed that daily highfrequency applications of TENS for 12 days with electrodes placed on the skin covering either ipsilateral or contra lateral paraspinal muscles reduced the development of mechanical hyperalgesia in a chronic constriction injury rats.

Similarly it also enlightened the fact that low-frequency nerve stimulation when applied to acupuncture points in the ipsilateral or contra lateral hind limbs reduced the development of thermal hyperalgesia, but only when TENS was delivered on the contra lateral side. Thus, once hyperalgesia develops, application of TENS to either the ipsilateral or the contra lateral hind limb is effective. One study was able to demonstrate the positive effects of TENS for relief of chronic musculoskeletal pain, and randomized controlled trials consistently demonstrate the effectiveness of TENS for intense, emergent, and postoperative nociceptive pain conditions.

However, the effectiveness of TENS is still controversial because of deficient and uninformative study designs. So, prolonged research of TENS machines, its theories and mechanisms and parameters are subject of being inadequate.

Therefore, the results outcome reflects that TENS is sufficiently associated with improvement on multiple outcome variables in addition to pain relief, for those who are using it for quite a long time. Also, for those who tried conventional pain relief methods and no good came out of it, long-term TENS use continues to be efficacious.

\section{References}

[1] NIH Public Access, Curr Rheumatol Rep. 2008 December; 10(6): 492-499. Published in final edited form as: Curr Rheumatol Rep. 2008 December; 10(6): 492-499.

[2] Somers DL, Clemente FR. Transcutaneous electrical nerve stimulation for the management of neuropathic pain: the effects of frequency and electrode position on prevention of allodynia in a rat model of complex regional pain syndrome type II. Phys Ther 2006;86:698-709. [PubMed: 16649893]

[3] TENS Machine, http://patient.info/in/health/tensmachines-leaflet.

[4] TENS by Chemelec, Available at: http://chemelec.com/Projects/Tens/

[5] Gate Theory, Available at: http://www.d.umn.edu/ jfitzake/Lectures/DMED/Somat osensation/Somatosensation/GateTheory.html

[6] TENS, Available at: https://en.wikipedia.org/wiki/Transcutaneous_electrical nerve_stimulation

[7] Square Wave, Available: https://en.wikipedia.org/wiki/Square_wave

[8] Sluka KA, Deacon M, Stibal A, et al. Spinal blockade of opioid receptors prevents the analgesia produced by TENS in arthritic rats. J Pharmacol Exp Ther 1999;289:840-846. [PubMed: 10215661]

[9] Sluka KA, Vance CG, Lisi TL. High-frequency, but not low-frequency, transcutaneous electrical nerve stimulation reduces aspartate and glutamate release in

\section{Volume 5 Issue 11, November 2016}




\section{International Journal of Science and Research (IJSR) \\ ISSN (Online): 2319-7064}

Index Copernicus Value (2015): 78.96 | Impact Factor (2015): 6.391

the spinal cord dorsal horn.

J Neurochem 2005;95:1794-1801. [PubMed: 16236028]• Of importance. High-frequency TENS reduces the release of excitatory neurotransmitters in the spinal cord through activation of $\delta$-opioid receptors.

[10]555 Timer, Wikipedia, Available at: https://en.wikipedia.org/wiki/555_timer_IC

[11] Electrotherapy, Wikipedia, Available: https://en.wikipedia.org/wiki/Electrotherapy

\section{Author Profile}

Soumyadeep Sarkar is currently pursuing B. Tech in Electrical and Electronics Engineering from Kurukshetra University, Kurukshetra. He is a research scholar and also an eminent member of The Institute of Electrical and Electronics Engineers (IEEE). His interested areas of research are Electrical Machines and Drives, flexible AC transmission systems, High Voltage AC and Power Electronics. His other research interests include Quantum Physics, Biomedical Science, Classical Mechanics, Astrophysics and Biological Psychiatry. 